University of Nebraska - Lincoln

DigitalCommons@University of Nebraska - Lincoln

$6-22-2007$

\title{
Thermal entanglement of two interacting qubits in a static magnetic field
}

G. Lagmago Kamta

Université de Sherbrooke, Quebec

Andrei Y. Istomin

University of Nebraska-Lincoln, aistomin2@unl.edu

Anthony F. Starace

University of Nebraska-Lincoln, astarace1@unl.edu

Follow this and additional works at: https://digitalcommons.unl.edu/physicsstarace

Part of the Physics Commons

Kamta, G. Lagmago; Istomin, Andrei Y.; and Starace, Anthony F., "Thermal entanglement of two interacting qubits in a static magnetic field" (2007). Anthony F. Starace Publications. 103.

https://digitalcommons.unl.edu/physicsstarace/103

This Article is brought to you for free and open access by the Research Papers in Physics and Astronomy at DigitalCommons@University of Nebraska - Lincoln. It has been accepted for inclusion in Anthony F. Starace Publications by an authorized administrator of DigitalCommons@University of Nebraska - Lincoln. 
Published in The European Physical Journal D 44 (2007) , pp. 389-400; DOI: 10.1140/epjd/e2007-00207-5 Copyright @ EDP Sciences, Società Italiana di Fisica, Springer-Verlag 2007. Used by permission. http://epjd.edpsciences.org/articles/epjd/pdf/2007/09/d07116.pdf

Submitted March 10, 2007; published online June 22, 2007.

\title{
Thermal entanglement of two interacting qubits in a static magnetic field
}

\author{
G. Lagmago Kamta ${ }^{1}$, A. Y. Istomin ${ }^{2, *}$, and A. F. Starace ${ }^{2, \dagger}$ \\ ${ }^{1}$ Laboratoire de Chimie Théorique, Faculté des Sciences, Université de Sherbrooke, Sherbrooke, Quebec J1K 2R1, Canada \\ ${ }^{2}$ Department of Physics and Astronomy, University of Nebraska-Lincoln, 116 Brace Laboratory, Lincoln, NE 68588-0111, USA \\ * Present address: Department of Computer Science and Bioinformatics Research Center, University of North Carolina, \\ 9201 University City Blvd., Charlotte, North Carolina 28223, USA \\ † Corresponding author: email astarace1@unl.edu
}

\begin{abstract}
We study systematically the entanglement of a two-qubit Heisenberg XY model in thermal equilibrium in the presence of an external arbitrarily-directed static magnetic field, thereby generalizing our prior work [G. Lagmago Kamta, A. F. Starace, Phys. Rev. Lett. 88, 107901 (2002)]. We show that a magnetic field having a component in the $x y-$ plane containing the spin-spin interaction components produces different entanglement for ferromagnetic (FM) and antiferromagnetic (AFM) couplings. In particular, quantum phase transitions induced by the magnetic field-driven level crossings always occur for the AFM-coupled qubits, but only occur in FM-coupled qubits when the coupling is of Ising type or when the magnetic field has a component perpendicular to the $x y$-plane. When the magnetic field has a component in the $x y$-plane, the cut-off temperature above which the entanglement of both the FM-and AFM-coupled qubits vanishes can always be controlled using the magnetic field for any value of the XY coupling anisotropy parameter. Thus, by adjusting the magnetic field, an entangled state of two spins can be produced at any finite temperature. Finally, we find that a higher level of entanglement is achieved when the in-plane component of the magnetic field is parallel to the direction in which the XY exchange coupling is smaller.
\end{abstract}

Keywords: Entanglement and quantum nonlocality, Quantum information, Entanglement production, characterization, and manipulation, Quantized spin models, Thermal/mixed state entanglement, Entanglement in spin models, Entanglement in quantum phase transitions

\section{Introduction}

Since the inception of quantum information science, quantum entanglement has been regarded as a physical resource for quantum information processing [1-4]. Potential applications of quantum entanglement have stimulated research on ways to quantify and measure entanglement in general [5-10], as well as on analyses of a number of quantum systems holding promise for practical realization of quantum information processing. Particular attention has been devoted to a variety of quantum systems consisting of a chain of particles whose interactions with each other can be modeled effectively by the Heisenberg spin-spin exchange coupling. The Heisenberg XYZ Hamiltonian for a chain of $N$ spin-1/2 particles with uniform nearest-neighbor interactions can be written as

$$
H_{0}=\sum_{n=1}^{N}\left(J_{x} S_{n}^{x} S_{n+1}^{x}+J_{y} S_{n}^{y} S_{n+1}^{y}+J_{z} S_{n}^{z} S_{n+1}^{z}\right)
$$

where $S_{n}^{\alpha}=1 / 2 \sigma_{n}^{\alpha}(\alpha=x, y, z)$ denotes the $\alpha$ th component of the local spin $1 \frac{1}{2}$ operator at site $n$, while $\sigma_{n}^{\alpha}$ is the corresponding Pauli matrix. The periodic boundary condition, $S_{N+1}=\mathrm{S}_{1}$, usually applies. The $J_{\alpha}$ are real coupling constants for the spin interaction; $J_{\alpha}>0$ for an antiferromagnetic (AFM) chain and $J_{\alpha}<0$ for a ferromagnetic (FM) chain.

The inter-qubit interactions in a number of systems can be described effectively by the Hamiltonian in equation (1). For instance, the isotropic Heisenberg coupling $\left(J_{x}=J_{y}=J_{z}\right)$ [11] and the transverse XY coupling [12] between electron spins in semiconductor quantum dots have been proposed for use in solid-state realizations of quantum computing, while the effective Ising-type coupling (e.g., $J_{y}=J_{z}=0$ ) between neutral atoms in periodic micropotentials has been proposed to create cluster states on which quantum information can be imprinted, processed, and read out by sequences of one-qubit 
measurements [13]. Hamiltonians similar to that in equation (1) but involving spin operators where $|S|$ is a large number effectively describe dipolar and RKKY couplings between magnetic nanodots $[14,15]$. Due to these and other potential applications, considerable attention has been devoted to the investigation of entanglement in systems described by the general Hamiltonian in equation (1). Indeed, general Hamiltonians that include Heisenberg spin-spin interactions have been proposed as "generic" [16] or "ideal" [17]

Many particular forms of the Hamiltonian in equation (1) have been studied theoretically in the literature, with the major focus being the influence of external parameters, such as the equilibrium temperature and external magnetic fields, on pure- and mixed-state entanglement of spin-chain systems (see, e.g., Refs. [18-30] and references therein). The universal properties of entanglement in $n$-spin ferromagnets were studied in reference [26]. The entanglement in isotropic, two-spin Heisenberg XXX (i.e., $J_{x}=J_{y}=J_{z}$ ) and XX (i.e., $J_{x}=J_{y}, J_{z}$ $=0$ ) models has been investigated in references $[19,20]$, respectively. The Heisenberg-Ising case $\left(J_{x}=J_{y}=0\right)$ with an arbitrary external magnetic field has been studied in reference [22]. The effect of an inhomogeneous magnetic field on the entanglement in isotropic, ferromagnetic Heisenberg two-qubit systems was studied in reference [27]. Global thermal entanglement of an XXZ-type Heisenberg chain in the presence of a uniform magnetic field was investigated in reference [30]. Entanglement of such a chain in an inhomogeneous magnetic field has been studied in reference [28] and the possibility of teleportation through a thermal equilibrium state of spins in such a chain was analyzed in reference [31]. Experimentally, the time-dependent Heisenberg-like interaction has been realized recently between ${ }^{1} \mathrm{H}$ and ${ }^{13} \mathrm{C}$ spins in a ${ }^{13} \mathrm{C}$-labeled chloroform molecule by means of NMR pulse sequences [32], thus evolving an initially unentangled pseudo-pure ground state to an entangled state and then to another factorized state via quantum phase transitions (QPTs).

Entanglement of two qubits coupled by an anisotropic Heisenberg XY coupling, which is the main subject of the present work, has received special attention, as the XY coupling can be realized in a variety of systems: (i) between electron spins in semiconductor quantum dots [12], (ii) between electrons in a 2D-electron gas within semiconductor-heterojunctions [33], and (iii) between neutral atoms in optical lattices [34]. Entanglement swapping between three pairs of spins coupled by a Heisenberg XY interaction has been used to create a three-qubit Greenberger-Horne-Zeilinger-like thermal state [35]. The influence of the magnetic field and the $\mathrm{XY}$ coupling anisotropy on the fidelity of quantum teleportation via one and two pairs of coupled qubits has been investigated in reference [36]. In the absence of an external magnetic field, entanglement studies have been carried out for a two-qubit anisotropic XY model (i.e., $J_{x} \neq J_{y}, J_{z}=0$ ) in references $[20,21]$. Chains involving more than two qubits have been considered in references [23,24,37], as well as in references $[19,22]$. It was shown in reference [20] that for a twoqubit $X Y$ chain in a transverse field $\mathbf{B}=B \hat{\mathbf{e}}_{z}$, a quantum phase transition occurs, and that there is a cutoff temperature above which the concurrence vanishes, independent of the value of the field (this is also the case for the XXX chain investigated in Reference [19]). For an antiferromagnetic two-qubit XY model with in-plane uniform or nonuniform magnetic fields, the value of the magnetic field at which the QPT occurs was found to be sensitive to the field orientation [38].

In a recent study of the two qubit Heisenberg XY chain in an external field $\mathbf{B}=B_{z} \hat{\mathbf{e}}_{z}$ [25], we have shown that the entanglement (i) is the same for FM and AFM chains and (ii) is independent of the sign of the anisotropy parameter $\gamma$ (i.e., is invariant upon permutation of the exchange coupling constants $J_{x}$ and $\left.J_{y}\right)$. We have also found that combining anisotropic spin exchange interactions with an external field, $\mathbf{B}=B_{z} \hat{\mathbf{e}}_{z}$, allows one to entangle two qubits at any finite temperature, $T$, by adjusting the field strength [25].

Here we present a detailed and largely analytic analysis of the entanglement of a two-qubit system coupled by a Heisenberg $\mathrm{XY}$ interaction and placed in an external uniform magnetic field whose magnitude and direction provide additional degrees of freedom to control the entanglement. We write the Hamiltonian for our system as follows:

$$
\begin{aligned}
\mathrm{H}= & \mathbf{B} \cdot\left(\mathbf{S}_{1}+\mathbf{S}_{2}\right)+2\left(J_{x} S_{1}^{x} S_{2}^{x}+J_{y} S_{1}^{y} S_{2}^{y}\right) \\
= & B_{-}\left(S_{1}^{+}+S_{2}^{+}\right)+B_{+}\left(S_{1}^{-}+S_{2}^{-}\right)+B_{z}\left(S_{1}^{z}+S_{2}^{z}\right) \\
& +J\left(S_{1}^{+} S_{2}^{-}+S_{1}^{-} S_{2}^{+}\right)+J \gamma\left(S_{1}^{+} S_{2}^{+}+S_{1}^{-} S_{2}^{-}\right),
\end{aligned}
$$

where $S^{ \pm}=S^{x} \pm i S^{y}$ denote the spin raising and lowering operators, and

$$
\begin{aligned}
& B_{ \pm}=\left(B_{x} \pm i B_{y}\right) / 2, J=\left(J_{x}+J_{y}\right) / 2, \\
& \gamma=\left(J_{x}-J_{y}\right) /\left(J_{x}+J_{y}\right) .
\end{aligned}
$$

The parameter $\gamma(-1 \leq \gamma \leq 1)$ measures the anisotropy of the spin coupling of the system; $\gamma=0$ for isotropic coupling, and $\gamma= \pm 1$ for Heisenberg-Ising couplings. We investigate here the most general case of entanglement of two qubits coupled by a Heisenberg XY exchange interaction in the presence of an external, arbitrarily-directed static magnetic field,

$$
\mathbf{B}=B_{x} \hat{\mathbf{e}}_{x}+B_{y} \hat{\mathbf{e}}_{y}+B_{z} \hat{\mathbf{e}}_{z},
$$

where $\hat{\mathbf{e}}_{i}(i=x, y, z)$ are the orthogonal unit vectors. First, we study the simplest cases, $\mathbf{B}=B_{x} \hat{\mathbf{e}}_{x}$ and $\mathbf{B}=B_{y} \hat{\mathbf{e}}_{y}$, for which we obtain analytic formulae for concurrence for an arbitrary value of the anisotropy parameter, $\gamma$. Second, we investigate cases when the magnetic field components lie in the $x z$-plane or in the $x y$-plane. Finally, we study the concurrence for a magnetic field whose direction is arbitrary and is characterized by two spherical angles. 


\section{The computational approach}

At thermal equilibrium at nonzero temperature, $T$, the generally mixed state of a Heisenberg spin chain is described by the density operator,

$$
\rho=Z^{-1} \exp \left(-H / k_{B} T\right)
$$

where $Z=\operatorname{Tr}\left[\exp \left(-H / k_{B} T\right)\right]$ is the partition function and $\mathrm{H}$ is the total Hamiltonian of the system, which includes $H_{0}$ and interactions with external fields.

In the present work, we quantify the pairwise entanglement of spin states in a Heisenberg chain by an established measure, the "entanglement of formation" [5]. For a bipartite pure state $|\Psi\rangle$, it is given by the von Neumann entropy of either of its two parts, i.e., $E_{F}(\Psi)=-\operatorname{Tr}\left(\rho \log _{2} \rho\right)$, where $\rho$ is the partial trace of $|\Psi \Psi|$ over either of the two systems. For a mixture of states with probabilities $p_{j}$, described by $\rho=\Sigma_{j} p_{j}\left|\Psi_{j}\right\rangle\langle$ $\Psi_{j} \mid$, the entanglement of formation is defined as $E_{F}(\rho)=$ min $\sum_{j} p_{j} E_{F}\left(\Psi_{j}\right)$. For a $2 \times 2$ system, the entanglement of formation can be written in analytical form as $[5,39,40]$

$$
E_{F}(\rho)=h\left(\frac{1+\sqrt{1-C^{2}}}{2}\right),
$$

where $h$ is the binary entropy function $h(x)=-x \log _{2} x-(1-$ $x) \log _{2}(1-x)$ and $C$ is the concurrence, given by

$$
C=\max \left\{0, \sqrt{\lambda}_{1}-\sqrt{\lambda}_{2}-\sqrt{\lambda}_{3}-\sqrt{\lambda}_{4}\right\},
$$

where the $\lambda_{i}(i=1,2,3,4)$ are the eigenvalues of the operator

$$
R=\rho\left(\sigma_{y} \otimes \sigma_{y}\right) \rho^{*}\left(\sigma_{y} \otimes \sigma_{y}\right),
$$

where $\lambda_{1}$ is the largest eigenvalue, and $\sigma_{y}$ denotes the usual Pauli matrix. Similarly to $E$, the concurrence $C$ ranges between 0 (no entanglement) and 1 (maximum entanglement), and is a monotonically increasing function of $E$, so that the concurrence $C$ itself is a measure of entanglement. As the density operator $\rho$ in equation (5) describes a thermal state, its entanglement is referred to as thermal entanglement [19]. We use the terms concurrence and entanglement throughout this paper to refer to thermal concurrence and thermal entanglement, respectively, unless otherwise stated.

In the basis $\{\varphi\} \equiv\{|00\rangle,|01\rangle,|10\rangle,|11\rangle\}$, where $|0\rangle$ denotes spin up and $|1\rangle$ denotes spin down, the Hamiltonian in equation (2) may be represented as

$$
\mathbf{H}=\left(\begin{array}{cccc}
B_{z} & B_{-} & B_{-} & \gamma J \\
B_{+} & 0 & J & B_{-} \\
B_{+} & J & 0 & B_{-} \\
\gamma J & B_{+} & B_{+} & -B_{z}
\end{array}\right)
$$

This Hermitian matrix is full, making it very difficult to obtain simple analytic expressions for eigenvalues and eigenvectors of $H$, for the density operator (5), as well as for the concur- rence $C$ (7). The eigenvalues $E$ of $H$ are solutions of the algebraic equation,

$$
\begin{aligned}
(E+J)\left[\left(B_{z}^{2}+\right.\right. & \left.J^{2} \gamma^{2}-E^{2}\right)(J-E) \\
& \left.-4 B_{-} B_{+} E-2 J \gamma\left(B_{-}^{2}+B_{+}^{2}\right)\right]=0,
\end{aligned}
$$

so that only one eigenvalue, $E=-J$, has a simple form. However, for special cases, such as $B_{z}=0$, simple analytic expressions for the eigenvalues and eigenvectors of $H$ may be obtained.

Obtaining analytic expressions for eigenvalues and eigenvectors of $H$ allows one to derive analytic expressions for the concurrence. A direct derivation of expressions for the $\lambda_{i}$ 's in equation (7) requires an analytical evaluation of the density matrix $\rho$, which is usually not trivial in the computational basis $\{\varphi\} \equiv\{|00\rangle,|01\rangle,|10\rangle,|11\rangle\}$. To obtain analytic expressions for the $\lambda_{i}$ 's when the eigenvalues and eigenvectors of $H$ are available, we use the following approach: let $\{\psi\}=$ $\left\{\left|\psi_{1}\right\rangle,\left|\psi_{2}\right\rangle,\left|\psi_{3}\right\rangle,\left|\psi_{4}\right\rangle\right\}$ denote a set of orthogonal eigenstates of $H$, where $\left|\psi_{j}\right\rangle(j=1,2,3,4)$ denotes the eigenvector associated with the eigenvalue $E_{j}$. The representation $\rho_{\psi}$ of $\rho$ in this basis is diagonal and is given by

$$
\left(\rho_{\psi}\right)_{j k}=\delta_{j, k} Z^{-1} \exp \left(-E_{j} / k_{B} T\right)
$$

The operator $S$ that transforms from the representation $\{\psi\}$ to $\{\varphi\}$ is unitary (i.e., $S S^{\dagger}=S^{\dagger} S=I$, where $I$ is the unit operator, and where $†$ denotes Hermitian conjugation), and is given by $S_{j k}=\left\langle\psi_{j} \mid \varphi_{k}\right\rangle$. Any operator $A$ in the $\{\varphi\}$-representation may be expressed in terms of its corresponding form $A_{\psi}$ in the $\{\psi\}$ representation by $A=S^{\dagger} A_{\psi} S$. Thus, the operator $R$, given by equation (8) in the computational basis, may be rewritten as

$$
\begin{aligned}
R & =S^{\dagger} \rho_{\psi} S S^{\dagger}\left(\sigma_{y} \otimes \sigma_{y}\right)_{\psi} S S^{\dagger} \rho_{\psi}^{*} S S^{\dagger}\left(\sigma_{y} \otimes \sigma_{y}\right)_{\psi} S \\
& \equiv S^{\dagger} R_{\psi} S,
\end{aligned}
$$

where $R_{\psi} \equiv \rho_{\psi}\left(\sigma_{y} \otimes \sigma_{y}\right)_{\psi} \rho_{\psi}^{*}\left(\sigma_{y} \otimes \sigma_{y}\right)_{\psi}$, and where $\left(\sigma_{y} \otimes \sigma_{y}\right)_{\psi}$ is the representation of the operator $\left(\sigma_{y} \otimes \sigma_{y}\right)$ in the $\{\psi\}$-basis. Since $R_{\psi}=S R S^{\dagger}$, the eigenvalues of $R$ in the basis $\{\varphi\}$ are identical to those of $R_{\psi}$ in the $\{\psi\}$-basis. Therefore, in order to obtain the $\lambda_{i}$ 's, one has to find the eigenvalues of $R_{\psi}$, which is easier to evaluate analytically [41] due to the diagonal structure of $\rho_{\psi}$, shown in equation (11).

For cases when the eigenvalues of $H$ cannot be obtained analytically, we evaluate the concurrence numerically in the standard basis as follows: the eigenvalues $E_{j}$ of $H$, and the corresponding eigenvectors $\left|\psi_{j}\right\rangle(j=1,2,3,4)$, are calculated using standard diagonalization routines. Then the eigenvectors are used to compute the transformation matrices $S$ and $S^{\dagger}$, and to derive the density matrix $\rho=S^{\dagger} \rho_{\psi} S$ from the density matrix $\rho_{\psi}$ given by equation (11). 


\section{Case of an external field $B=B_{x} \hat{\mathbf{e}}_{x}$}

The solution of the eigenvalue problem for the Hamiltonian matrix (9) for $\mathbf{B}=B_{x} \hat{\mathbf{e}}_{x}$ yields

$$
E_{1}=-\gamma J, E_{2}=-J, E_{3,4}=[(1+\gamma) J \pm \eta x] / 2,
$$

and the corresponding eigenvectors are

$$
\begin{aligned}
& \left|\psi_{1}\right\rangle=\left|\Phi^{-}\right\rangle, \quad\left|\psi_{2}\right\rangle=\left|\Psi^{-}\right\rangle, \\
& \left|\psi_{3,4}\right\rangle=N_{3,4}\left[B_{x}\left|\Phi^{+}\right\rangle+\left(E_{3,4}-\gamma J\right)\left|\Psi^{+}\right\rangle\right],
\end{aligned}
$$

where $\eta_{x}=\left((1-\gamma)^{2} J^{2}+4 B_{x}^{2}\right)^{1 / 2}$. The normalization constants are given by $N_{3,4}=\left(2 /\left[\eta_{x}\left(\eta_{x} \pm J(1-\gamma)\right)\right]\right)^{1 / 2}$. Parameters having the index " 3 " take the " + " sign, while those having the index "4" take the “-” sign. $\left|\Phi^{ \pm}\right\rangle$and $\left|\Psi^{ \pm}\right\rangle$denote the four maximally entangled Bell states:

$$
\left|\Phi^{ \pm}\right\rangle=\frac{1}{\sqrt{2}}(|00\rangle \pm|11\rangle), \quad\left|\Psi^{ \pm}\right\rangle=\frac{1}{\sqrt{2}}(|01\rangle \pm|10\rangle) .
$$

Since the eigenvectors in equation (14) are real, so is the transformation matrix $S$,

$$
S=\frac{1}{\sqrt{2}}\left(\begin{array}{cccc}
1 & 0 & 0 & -1 \\
0 & 1 & -1 & 0 \\
B_{x} N_{3} & N_{3}\left(E_{3}-\gamma J\right) & N_{3}\left(E_{3}-\gamma J\right) & B_{x} N_{3} \\
B_{x} N_{4} & N_{4}\left(E_{4}-\gamma J\right) & N_{4}\left(E_{4}-\gamma J\right) & B_{x} N_{4}
\end{array}\right)
$$

Therefore, $R_{\psi}=\rho_{\psi}\left(\sigma_{y} \otimes \sigma_{y}\right)_{\psi} \rho_{\psi}\left(\sigma_{y} \otimes \sigma_{y}\right)_{\psi}$. As described in Section 2 we evaluate the concurrence (i.e., the $\lambda_{i}$ 's) by finding the eigenvalues of $R_{\psi}$ in the eigenstate basis $\{\psi\}$, in which the operator $\left(\sigma^{y} \otimes \sigma^{y}\right)$ is given by

$$
\left(\sigma^{y} \otimes \sigma^{y}\right)_{\psi}=\left(\begin{array}{cccc}
1 & 0 & 0 & 0 \\
0 & -1 & 0 & 0 \\
0 & 0 & J(1-\gamma) / \eta_{x} & -2 B_{x} / \eta_{x} \\
0 & 0 & -2 B_{x} / \eta_{x} & -J(1-\gamma) / \eta_{x}
\end{array}\right) .
$$

After lengthy calculations, one finds that the $\lambda_{i}$ 's are given by

$$
\begin{gathered}
\lambda_{1}=Z^{-2} \exp \left(2 \gamma J / k_{B} T\right), \\
\lambda_{2}=Z^{-2} \exp \left(2 J / k_{B} T\right), \\
\lambda_{3,4}=\frac{e^{-J(1+\gamma) / k_{B} T}}{Z^{2}}\left\{F_{\gamma, J}\left(B_{x}, T\right) \pm \sqrt{F_{\gamma, J}\left(B_{x}, T\right)^{2}-1}\right\},
\end{gathered}
$$

where

$$
\begin{gathered}
F_{\gamma, J}\left(B_{x}, T\right)=\left[\frac{4 B_{x}^{2}}{\eta_{x}^{2}}+\frac{(1-\gamma)^{2} J^{2}}{\eta_{x}^{2}} \cosh \left(\frac{\eta_{x}}{k_{B} T}\right)\right], \\
Z=\exp \left(\frac{J}{k_{B} T}\right)+\exp \left(-\frac{(1+\gamma) J+\eta_{x}}{2 k_{B} T}\right) \\
\quad+\exp \left(\frac{\gamma J}{k_{B} T}\right)+\exp \left(-\frac{(1+\gamma) J-\eta_{x}}{2 k_{B} T}\right),
\end{gathered}
$$

and where in $\lambda_{3}$ and $\lambda_{4}$ (cf. Equation (20)), “+” and “-”signs respectively are assumed.

The $\lambda_{i}$ 's in equations (18-20) cannot be ordered unless one assigns specific values to the parameters involved. This pre- vents one from writing a more specific analytic formula for the concurrence other than equation (7) with the $\lambda_{i}$ 's given by equations (18-20). In practice, we first evaluate the $\lambda_{i}$ 's, then order them by magnitude, with $\lambda_{1}$ being the largest, and then use equation (7) to derive the concurrence.

Interesting properties of the concurrence can readily be derived from equations (18-20): (i) for the anisotropy parameter $\gamma=1$ (i.e., $J_{y}=0$ ), the entanglement vanishes for both FM and AFM chains. In fact, the two terms in the corresponding Hamiltonian $H=B_{x}\left(S_{1}^{x}+S_{2}^{x}\right)+2 J_{x} S_{1}^{x} S_{2}^{x}$, both tend to impose spin order along the $x$-axis; (ii) for arbitrary $\gamma$ the concurrences for the FM and AFM chains are different, because the set of $\lambda_{i}$ 's is not invariant under the transformation $J \rightarrow-J$. However, for the specific case $\gamma=-1$ (i.e., $J_{x}=0$ ), the transformation $J \rightarrow-J$ leaves the set of $\lambda_{i}$ 's unchanged, thereby indicating that for $\gamma=-1$, the entanglement of the two qubits is the same for FM and AFM chains. This is the case of two qubits with Ising interaction in the presence of an orthogonal external magnetic field, which was studied in reference [22]; (iii) the set of $\lambda_{i}$ 's is not invariant under the transformation $\gamma \rightarrow-\gamma$, which corresponds to the permutation of $J_{x}$ and $J_{y}$, thereby indicating that the concurrence changes with the sign of the anisotropy parameter. Note that this contrasts with the $\mathbf{B}=B_{z} \hat{\mathbf{e}}_{z}$ case [25], where the concurrence is the same for FM and AFM chains, and is unchanged by a change in the sign of the anisotropy parameter; (iv) finally, and similarly to the $\mathbf{B}$ $=B_{z} \hat{\mathbf{e}}_{z}$ case in reference [25], the $\lambda_{i}$ 's in equations (18-20) are invariant under the transformation $B_{x} \rightarrow-B_{x}$, which means that the concurrence does not depend on the orientation of the B field along the $x$-axis, but only on its magnitude. As mentioned above, the case $\gamma=1$ is trivial, and the case $\gamma=-1$ has been studied [22]; therefore our discussion below will be restricted to values of $\gamma$ in the range $-1<\gamma<1$.

Consider first the isotropic Heisenberg chain, for which $\gamma$ $=0$. The concurrence obtained for this case is plotted with respect to $k_{B} T$ and $\left|B_{x}\right|$ in Figure $1 \mathrm{~b}$ for the AFM chain with $J=$ 1 , and in Figure 1c for the FM chain with $J=-1$. For comparison, we show similar results for the isotropic Heisenberg chain in Figure 1a for $\mathbf{B}=B_{z} \hat{\mathbf{e}}_{z}$ with $|J|=1$ [20,25]. In this case FM and AFM chains have the same concurrence, which vanishes identically for a temperature larger that the cutoff value $T_{C}$ given by $k_{B} T_{C} \equiv 1.1346 J$ [20].

In general, the plots in Figure 1 indicate that the concurrence is maximal at $T=0$, that it decreases with increasing temperature (owing to the increasing mixture of the ground state with other states), and that there is a cutoff temperature above which the concurrence vanishes. However, one sees that for the case $\mathbf{B}=B_{x} \mathbf{e}_{x}$, the cutoff temperature $T_{c}$ above which the concurrence vanishes depends on the magnitude of the $\mathbf{B}$ field. Figure 2 shows this cutoff temperature as a function of the magnitude $\left|B_{j}\right|(j=x, z)$, for external magnetic fields $\mathbf{B}=$ $B_{j} e_{j}$. For $\mathbf{B}=B_{z} \mathbf{e}_{z}$, the cutoff temperature is constant for both FM and AFM couplings. For $\mathbf{B}=B_{x} \mathbf{e}_{x}$ for the case of FM coupling, $T_{C}$ increases monotonically with $\left|B_{x}\right|$. In contrast, 

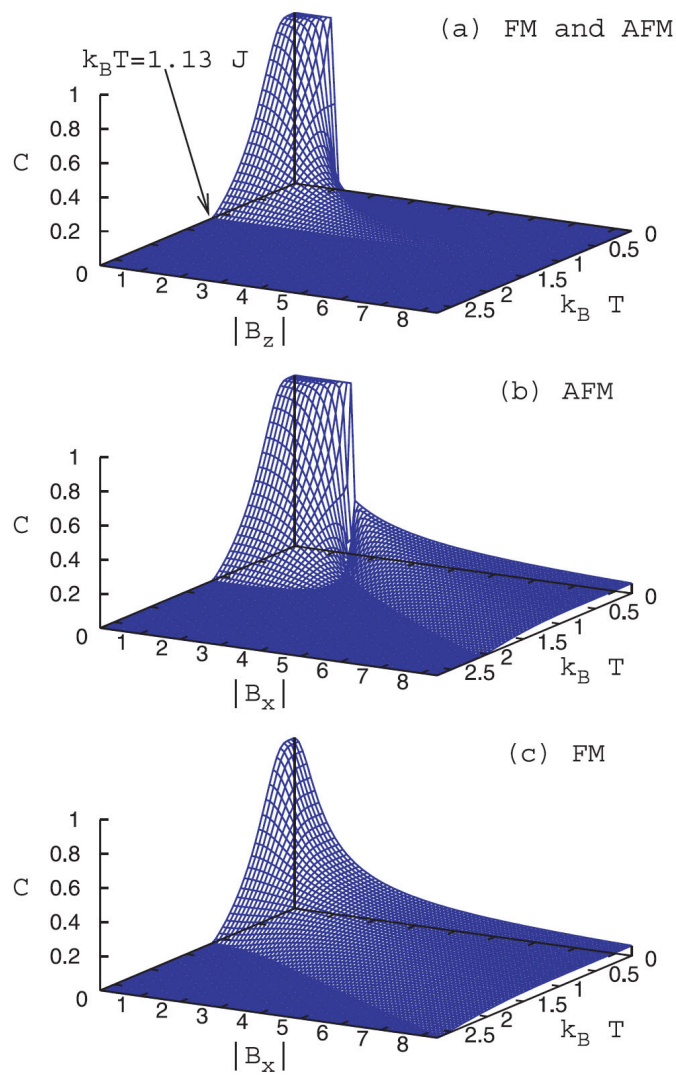

Figure 1. Comparison of the concurrences for the isotropic XY chain of two qubits as a function of $k_{B} T$ and the magnitude of the magnetic field $\mathbf{B}$ for two different directions of B. (a) FM and AFM chains ( $|J|$ $=1$ ) for $\mathbf{B}=B_{z} \mathbf{e}_{z}$; (b) AFM chain $(J=1)$ for $\mathbf{B}=B_{x} \mathbf{e}_{x}$; (c) FM chain $(J$ $=-1$ ) for $\mathbf{B}=B_{x} \mathbf{e}_{x}$.

the behavior of $T_{c}$ for the AFM coupled qubits in a $\mathbf{B}=B_{x} \mathbf{e}_{x}$ magnetic field displays two regions: a region corresponding to small values of $\left|B_{x}\right|$ up to approximately $\sqrt{2} J$, where $T_{C}$ decreases with $\left|B_{x}\right|$, and a region corresponding to values of $\left|B_{x}\right|$ larger than $\sqrt{2} J$, over which $T_{C}$ increases monotonically with $B_{x}$. (The origin of the quantity $\approx \sqrt{2} J$ is discussed later.) This means that for an isotropic XY chain of two qubits, by having an external magnetic field along the $x$-direction (i.e., in the plane of the spin coupling) instead of the z-direction (i.e., perpendicular to the plane of the spin coupling), one can induce entanglement between otherwise unentangled qubits at any temperature, by increasing the magnitude of the magnetic field $\mathbf{B}=B_{x_{x^{*}}} \mathbf{e}$ (As we have recently shown [25], generating spin entanglement in a two-qubit Heisenberg chain with an external field $\mathbf{B}=B_{z} \hat{\mathbf{e}}_{z}$ at any temperature is possible only for anisotropic exchange coupling.) Note that for the AFM case with $\mathbf{B}=B_{x} \mathbf{e}_{x}$, for $0.3 \lesssim k_{B} T \lesssim 1.2$ there is a range of magnetic field values beginning in the vicinity of $B_{x} \approx 2$ over which the concurrence is zero, but is nonzero for both lower and higher magnetic field values. In other words, the increase of the magnetic field in this case induces a revival of the concurrence.

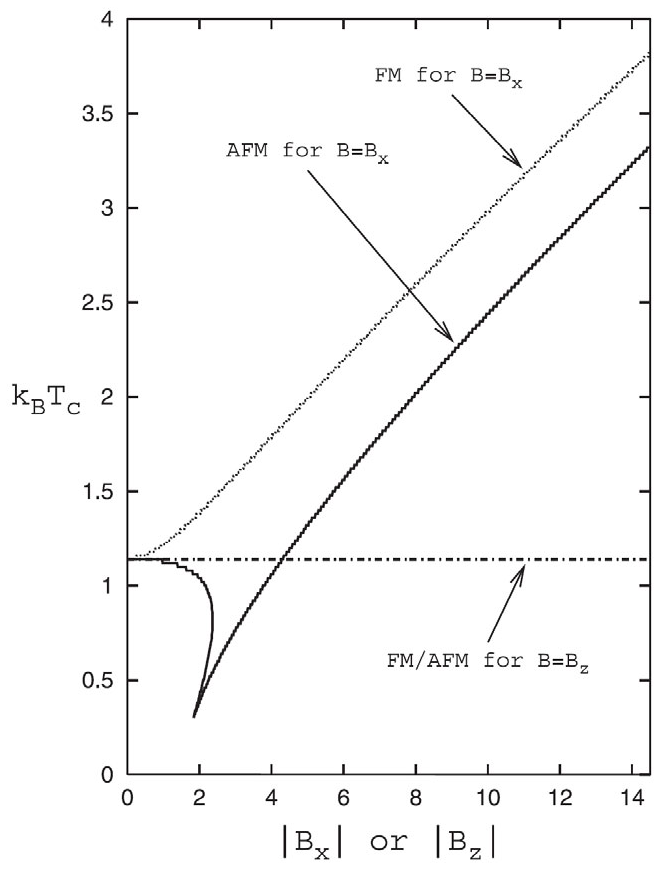

Figure 2. Cutoff temperature $T_{c}$ at which the concurrence $C$ for an isotropic $X Y$ chain of two qubits vanishes, plotted versus the magnitude of the magnetic field $\left|B_{j}\right|(j=x, z)$ : (i) FM and AFM chains with $\mathbf{B}=B_{z} \mathbf{e}_{z}$ (horizontal dot-dashed line); (ii) AFM chain with $\mathbf{B}=$ $B_{x} \mathbf{e}_{x}$ (solid curve); (iii) FM chain with $\mathbf{B}=B_{x} \mathbf{e}_{x}$ (dashed curve). In all cases, $|J|=1$.

Results for $\mathbf{B}=B_{x} \mathbf{e}_{X}$ and for various values of the anisotropy parameter are shown in Figure 3 for the FM case $(J=$ $-1)$ and in Figure 4 for the AFM case $(J=1)$. These plots also show a maximum concurrence at $T=0$, which decreases with increasing temperature and which vanishes when a cutoff temperature is reached. This cutoff temperature increases with the field strength, so that for any finite temperature one can always generate entanglement by increasing the field strength. One sees that in general, for a given value of $\left|B_{x}\right|$, the cutoff temperature increases with decreasing anisotropy parameter $\gamma$ (i.e., with increasing $J_{y}$ ). This means that one obtains higher cutoff temperatures by increasing the spin exchange coupling in the direction perpendicular to the $\mathbf{B}$ field. One can show that for asymptotically large $\left|B_{x}\right|$, i.e., for $\left|B_{x}\right| \gg J$ and $\left|B_{x}\right| \gg$ $\gamma$, the cutoff temperature $T_{C}$ above which the concurrence vanishes is given by

$$
k_{B} T_{c}=\frac{\left|B_{x}\right|}{\ln \left(\frac{4\left|B_{x}\right|}{|J||1-\gamma|}-2\right)},
$$

which clearly illustrates the increase of the cutoff temperature with decreasing anisotropy parameter $\gamma$.

The concurrence for the FM chain in Figure 1c varies continuously as a function of the magnetic field. This is not the case for the AFM chain in Figure $1 \mathrm{~b}$ at $T=0$, where the concurrence first equals unity as $B_{x}$ increases from zero, then drops sharply and discontinuously when $B_{x}$ reaches a critical 

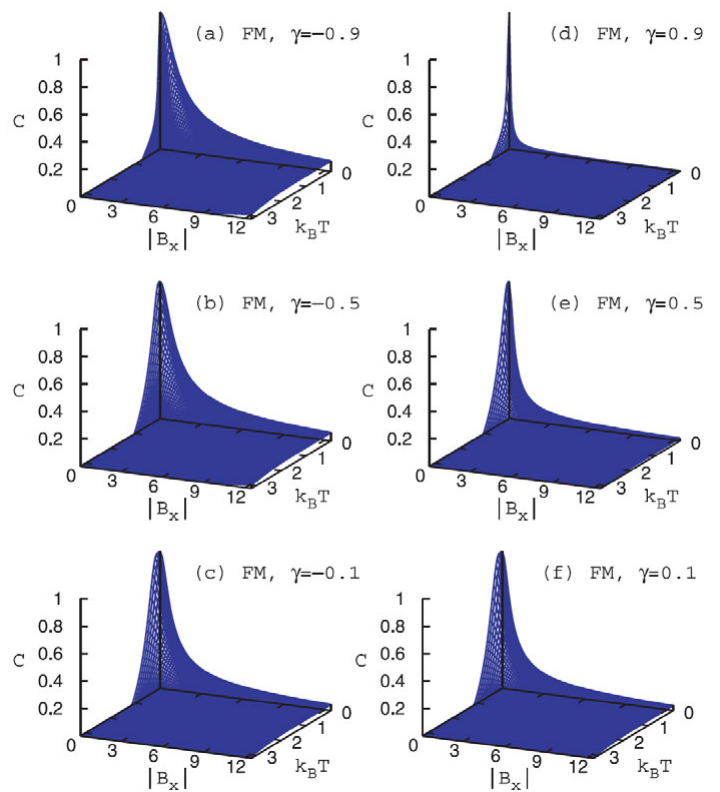

Figure 3. Concurrence of two qubits coupled by XY ferromagnetic coupling $(J=-1)$ as a function of $k_{B} T$ and the magnitude of the magnetic field $\mathbf{B}=B_{x} \mathbf{e}_{x}$ for six values of the anisotropy parameter $\gamma$, as indicated in each figure.
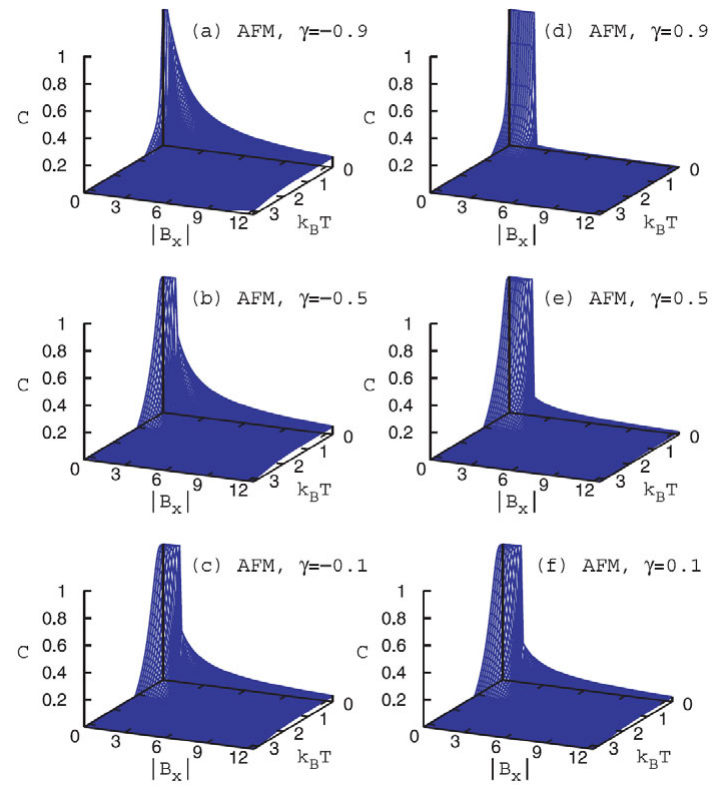

Figure 4. Same as Figure 3, but for the case of antiferromagnetic coupling $(J=1)$.

value $B_{x}^{\mathrm{c}}$. This nonanalytic behavior of the concurrence is due to the occurrence of a quantum phase transition (QPT) [42]. Similarly, plots of the concurrence for various values of the anisotropy parameter in Figures 3 and 4 indicate that a nonanalyticity occurs at $T=0$ for an AFM chain (see Figure 4), but never occurs for the FM chain (see Figure 3). In fact, taking the limit of the $\lambda_{i}$ 's in equations (18-20) for $T \rightarrow 0$, one finds that at $T=0$, the concurrence is given by

$$
C_{T=0}=(1-\gamma) \frac{|J|}{\eta_{x}}
$$

for the FM chain, and by

$$
C_{T=0}= \begin{cases}1 & \text { for }\left|B_{x}\right|<\sqrt{2(1+\gamma)} J, \\ \frac{1-(1-\gamma) J / \eta_{x}}{2} & \text { for }\left|B_{x}\right|=\sqrt{2(1+\gamma)} J, \\ (1-\gamma) J / \eta_{x} & \text { for }\left|B_{x}\right|>\sqrt{2(1+\gamma)} J\end{cases}
$$

for the AFM chain. Note that equations (23) and (24) hold for $-1<\gamma<1$. As mentioned above, the concurrence is identical for FM and AFM chains when $\gamma=-1$; in that case, the concurrence at $T=0$ is given by equation (24). Therefore, the concurrence for the AFM chain at $T=0$, in contrast to the FM chain, becomes a non analytic function of $\left|B_{x}\right|$ when $\left|B_{x}\right|$ reaches the critical value $B_{x}^{\mathrm{c}} \equiv(2(1+\gamma))^{1 / 2} J$ [or, equivalently, when $\eta$ reaches the critical value $\left.\eta_{x}^{\mathrm{c}}=(3+\gamma) J\right]$.

This QPT originates from a level-crossing, i.e., when an excited state energy becomes equal and then drops below a ground state energy, as the system parameter is varied [42]. This may occur for the AFM chain, but not for the FM chain, as illustrated in Figure 5 for $\gamma=0.5$. For a FM chain $(J<0)$, the state $\left|\psi_{4}\right\rangle$ has the lowest energy $E_{4}=-|J|$ for $\left|B_{X}\right|=0$. Increasing $\left|B_{\chi}\right|$ only lowers $E_{4}$ and further increases the gap between $E_{4}$ and the energy of the lowest excited state, so that $\left|\psi_{4}\right\rangle$ always remains the ground state of the FM two-qubit system. In contrast, for a two-qubit AFM system, the state $\left|\psi_{2}\right\rangle$ has its lowest energy $E_{2}=-J$ for $\left|B_{x}\right|=0$. As $\left|B_{x}\right|$ increases, $E_{4}$ decreases and becomes equal to $E_{4}=-J=E_{2}$ when $B_{x}$ reaches the critical magnetic field $B_{x}^{\mathrm{c}}$. As $\left|B_{x}\right|$ increases beyond $B_{x}^{\mathrm{c}}$, a level crossing occurs as $\left|\psi_{4}\right\rangle$ becomes the ground state of the AFM chain.

It follows that at $T=0$, the FM system is always in its ground state $\left|\psi_{4}\right\rangle$, and computing the concurrence for this pure state leads to equation (23). The level crossing induced by the external magnetic field in the AFM chain is the source of the non-analyticity of the concurrence. Indeed, for $\left|B_{x}\right|<$ $\left|B_{x}^{\mathrm{C}}\right|$, the system is in the pure state $\left|\psi_{2}\right\rangle$ whose entanglement is 1. For $\left|B_{x}\right|=B_{x}^{\mathrm{c}},\left|\psi_{2}\right\rangle$ and $\left|\psi_{4}\right\rangle$ have the same (lowest) energy, the ground state is therefore an equal mixture of these two states, whose entanglement is given by the second equation in equation (24). To show this, one can use the density operator $\rho=1 / 2\left|\psi_{2}\right\rangle\left\langle\psi_{2}|+1 / 2| \psi_{4}\right\rangle\left\langle\psi_{4}\right|$, which describes this mixture. Finally, for $\left|B_{x}\right|>B_{x}^{c}$, the AFM chain at $T=0$ is in the pure state $\left|\psi_{4}\right\rangle$, whose entanglement is given by the last equation in equation (24).

\section{Case of an external field $B=B_{y} \mathbf{e}_{y}$}

The isotropic Heisenberg XY chain has rotational symmetry about the $z$-axis. The concurrence is therefore the same for all orientations of the external magnetic field perpendicular to the $z$-axis. In other words, for the isotropic Heisenberg chain, the entanglement of the two qubits in the presence of an external magnetic field $\mathbf{B}=B_{y} \mathbf{e}_{y}$ is the same as for the case $\mathbf{B}=B_{x} \mathbf{e}_{x}$ investigated in the previous section. 

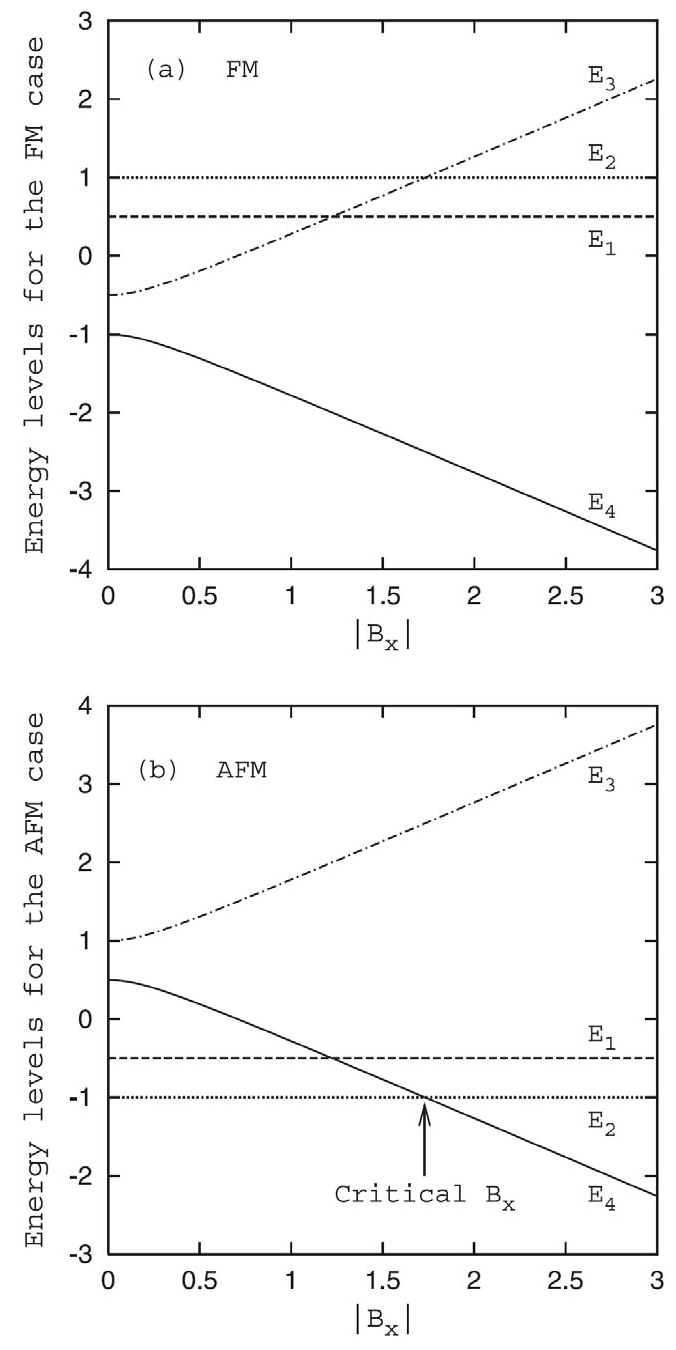

Figure 5. Energy levels of the FM (top plot) and of the AFM (bottom plot) $X Y$ chains of the two qubits, vs the magnitude $\left|B_{x}\right|$ of the external magnetic field $\mathbf{B}=B_{X} \mathbf{e}_{x}$. Energies and $\left|B_{X}\right|$ are given in units of $J$. Results shown are obtained with $\gamma=0.5$. $E_{j}$ denotes the energy of the eigenstate $\Psi_{j}, j=1,2,3$, 4. A level crossing occurs at the critical magnetic field for the AFM chain but not for the FM chain.

For the anisotropic Heisenberg XY chain (i.e., $\gamma \neq 0$ ), the rotational symmetry about the $z$-axis is broken, so that the cases $\mathbf{B}=B_{x} \mathbf{e}_{x}$ and $\mathbf{B}=B_{y} \mathbf{e}_{y}$ would lead to different concurrences. However, the Hamiltonians (2) for these two cases can be derived from one another by a permutation of the $x$ and $y$ axes. Therefore, the concurrence for $\mathbf{B}=B_{y} \mathbf{e}_{y}$ can be deduced from the concurrence for $\mathbf{B}=B_{x} \mathbf{e}_{x}$ using the transformations $B_{x} \rightarrow B_{y}, J_{x} \rightarrow J_{y}$, and $\gamma \rightarrow-\gamma$. Explicitly, this means that the $\lambda_{i}$,s leading to the concurrence for $\mathbf{B}=B_{y} \mathbf{e}_{y}$ are given by equations (18-21), with $B_{x}$ replaced by $B_{y}$ and $\gamma$ by $-\gamma$. Therefore, the plots shown in Figures 1-5 are also valid for the case $\mathbf{B}=$ $B_{y} \mathbf{e}_{y}$, provided that $B_{x}$ is replaced by $B_{y}$ and the corresponding $\gamma$ by $-\gamma$. For example, all plots in Figure 3 (Figure 4) represent the concurrence for the FM XY chain (AFM XY chain) in an external field $\mathbf{B}=B_{y} \mathbf{e}_{y}$, with plots labelled (a), (b), (c), (d), (e) and (f) corresponding to $\gamma=0.9, \gamma=0.5, \gamma=0.1, \gamma=$ $-0.9, \gamma=-0.5$ and $\gamma=-0.1$, respectively.

The concurrence at $T=0$ for $\mathbf{B}=B_{y} \mathbf{e}_{y}$ is also given by equations (23) for the FM chain and by equation (24) for the AFM chain, with $B_{x}$ replaced by $B_{y}$ and $\gamma$ replaced by $-\gamma$. It follows that in the presence of an external magnetic field $\mathbf{B}=$ $B_{y} \mathbf{e}_{y}$ a quantum phase transition occurs for the AFM chain but not for the FM chain, and that the cutoff temperature above which the concurrence vanishes increases with $B_{y}$, so that one can entangle the two spins at any temperature by adjusting the magnetic field. Also, one sees that for given $\left|B_{y}\right|$ one obtains larger cutoff temperatures by increasing the anisotropy parameter $\gamma$, i.e., by increasing the exchange coupling $J_{x}$ in the direction perpendicular to the $\mathbf{B}$ field.

\section{Case of an external field $B=B_{x} e_{x}+B_{y} \mathbf{e}_{y}$}

With this configuration of the external magnetic field, the eigenvalues and eigenvectors of the Hamiltonian in equation (9) have complicated analytic forms (cf. Sect. 7), thereby preventing us from obtaining analytical expressions for the $\lambda_{i}$ 's using the eigenstate basis approach. We rather use the numerical approach to evaluate the concurrence for this case. Figure 6 displays the concurrence with respect to the $x$ - and $y$-components of the $\mathbf{B}$ field, for various anisotropy parameters at $T=0$. We present only results for $\gamma \geq 0$ because the transformation $\gamma \rightarrow$ $-\gamma$ followed by a permutation of the $x$ - and $y$-axes leaves the system unchanged, i.e., $C\left(\gamma, B_{x}, B_{y}\right)=C\left(-\gamma, B_{y}, B_{x}\right)$. Thus, from each plot in Figure 6 for a given $\gamma$, the corresponding plot for the anisotropy parameter $-\gamma$ is deduced by making a permutation of $B_{x}$ and $B_{y}$. One sees that the concurrence is invariant under any of the transformations $B_{x} \rightarrow-B_{x}$ and $B_{y} \rightarrow-B_{y}$.

Figures 6a and 6e show the concurrence for the isotropic chain $(\gamma=0)$, and illustrate the symmetry of the concurrence with respect to the axis through the origin and perpendicular to the $\left(B_{x}, B_{y}\right)$ plane. This consequence of the rotational symmetry of the isotropic Heisenberg XY chain about the z-axis suggests that there is no privileged direction for entanglement. In fact, due to this symmetry, the present orientation of the magnetic field for $\gamma=0$ leads to the same concurrence as for the cases $\mathbf{B}=B_{x} \mathbf{e}_{x}$ discussed in detail in Section 3. In other words, for the $\mathbf{B}=B_{x} \mathbf{e}_{x}+B_{y} \mathbf{e}_{y}$ case with isotropic exchange interaction, the $\lambda_{i}$ 's are given by equations (18-20), and the concurrence at $T=0$ is given by equations (23) and (24), in which $B_{x}$ (resp. $\left.\eta_{x}\right)$ is replaced by $B_{x y} \equiv\left(B_{x}^{2}+B_{y}^{2}\right)^{1 / 2}$ (resp. $\eta_{x y}=\left(J^{2}\right.$ $\left.\left.+4 B_{x y}^{2}\right)^{1 / 2}\right)$, with $\gamma=0$. Therefore, at $T=0$, no quantum phase transition occurs for the FM chain, and $C=J / \eta_{x y}$. This is confirmed by the numerical results in Figure 6a, which show a continuous decrease of the concurrence with increasing $B_{x y}$. On the other hand, for the isotropic chain at $T=0$, a quantum phase transition occurs for the AFM chain when $B_{x y}$ reaches the critical value $\sqrt{2} J$, i.e., when $B_{x}^{2}+B_{y}^{2}=2 J^{2}$, which describes a circle of radius $\sqrt{2} J$, centered at the origin in the 

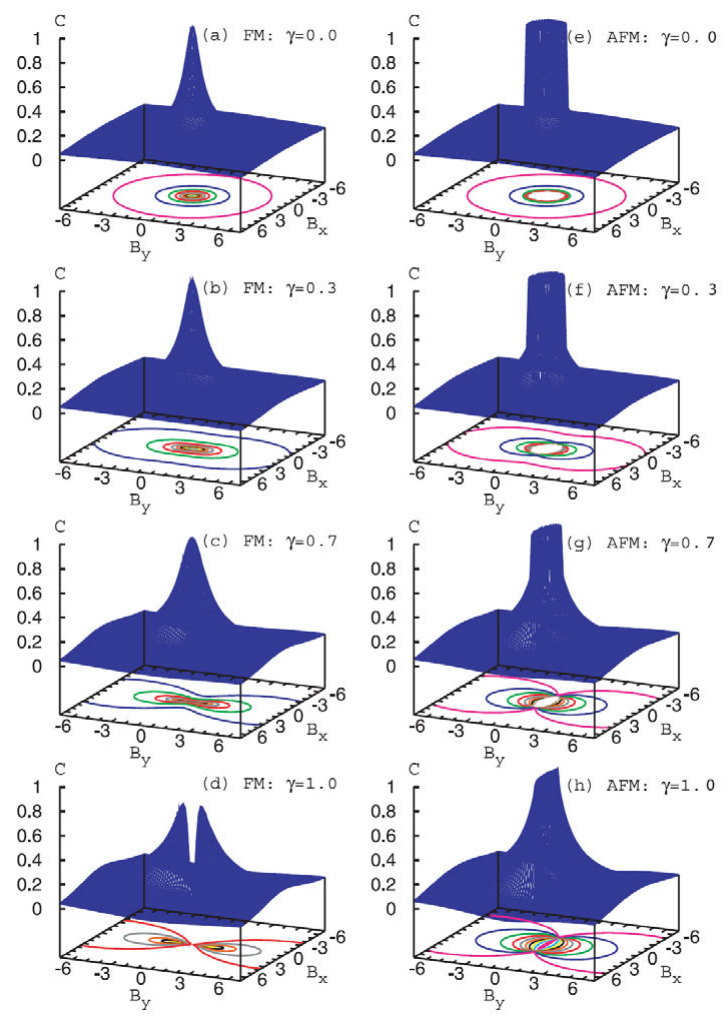

Figure 6. Concurrence of two qubits coupled by Heisenberg FM and AFM interactions for $|J|=1.0$ at zero temperature, $T=0$, in the presence of an external in-plane magnetic field, $\mathbf{B}=B_{x} \mathbf{e}_{x}+B_{y} \mathbf{e}_{y}$ for four values of the anisotropy parameter $\gamma: 0,0.3,0.7$, and 1.0. Note that for $\gamma=1.0$ in (d) and (h), one has $C \equiv 0$ along the line $B_{y}=0$.

$\left(B_{x}, B_{y}\right)$ plane. This is confirmed by the numerical results in Figure 6e, which show that the concurrence is equal to unity for $B_{x}$ and $B_{y}$ within a circular disk of radius $\sqrt{2} J$ centered at the origin; when $B_{x}$ and $B_{y}$ increase so that they are both located on the circle of radius $\sqrt{2} J$ (i.e., at the rim of the disk of radius $\sqrt{2} J$ ), the concurrence decreases sharply to $1 / 3$; finally, for $B_{x}$ and $B_{y}$ outside the disk of radius $\sqrt{2} J$, the concurrence decreases analytically as $C=J / \eta_{x y}$. Figure 6a for the FM chain shows that the concurrence is maximal $(C=1)$ for $\mathbf{B}=$ 0 , and decreases analytically with increasing $B_{x y}$ as $C=J / \eta_{x y}$.

The anisotropic cases with $\gamma>0$ (i.e. $J_{x}>J_{y}$ ) shown in Figure 6 illustrate the breakdown of the rotational symmetry about the $z$-axis. With increasing $\gamma$, the concurrence for the FM chain stretches along the $y$-axis, so that the maximum entanglement is achieved for a $\mathbf{B}$ field directed along the $y$-axis. For the AFM chains, the circular disk in the $\left(B_{x}, B_{y}\right)$ plane, which corresponds to the maximum entanglement for $\gamma=$ 0 , becomes elliptical, with the major axis along the $x$-direction and the minor axis along the $y$-direction. With increasing anisotropy, the major axis of the ellipse increases, while the minor axis decreases. For the AFM chain, having a $\mathbf{B}$ field increasingly aligned along the $y$-axis also maximizes the concurrence, which is less stretched along the $y$-axis than for the FM chain. From the property $C\left(\gamma, B_{x}, B_{y}\right)=C\left(-\gamma, B_{y}, B_{x}\right)$ mentioned above, it appears that for $\gamma<0$ (i.e. $J_{y}>J_{x}$ ), the max-
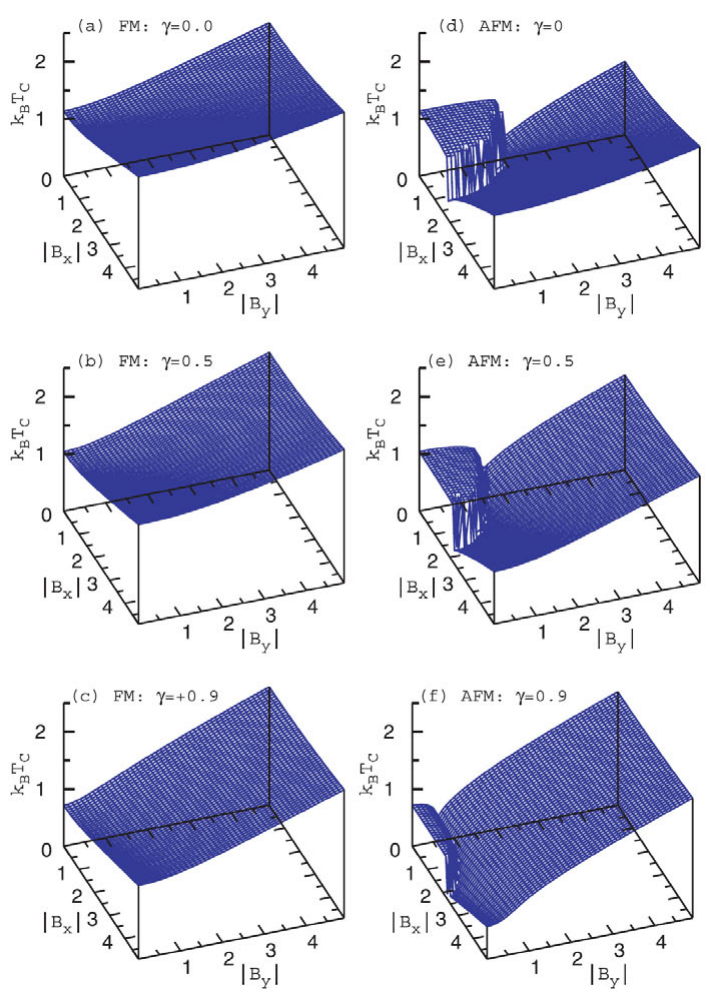

Figure 7. The cutoff temperature above which the concurrence vanishes for $\mathbf{B}=B_{x} \mathbf{e}_{x}+B_{y} \mathbf{e}_{y}$ as a function of $B_{x}$ and $B_{y}$ for various asymmetry parameters $\gamma$.

imum entanglement is obtained for a $\mathbf{B}$ field increasingly aligned along the $x$-axis. Therefore, one may conclude that in general, maximum entanglement is achieved with an external B field aligned in the direction parallel to the axis in which the exchange coupling is smaller. It follows from our results for $\gamma=1$ in Section 3 that for $\mathbf{B}=B_{x} \mathbf{e}_{x}$, the concurrence vanishes identically. Therefore, in Figures $6 \mathrm{~d}$ and $6 \mathrm{~h}$ the concurrence is zero along the line $B_{y}=0$.

With increasing temperature, the concurrence decreases as a result of a mixture of the ground state with excited states, and there is always a cutoff temperature, $T_{c}$, above which the concurrence vanishes. This cutoff temperature is plotted in Figure 7 as a function of $B_{x}$ and $B_{y}$ for various values of the anisotropy parameter $\gamma$. For the FM case (Figures $7 \mathrm{a}-7 \mathrm{c}$ ), $T_{c}$ increases monotonically with both $B_{x}$ and $B_{y}$, thereby indicating that increasing the field strength generates entanglement between otherwise unentangled spins. This is true independently of the anisotropy parameter. Note that the influence of the $y$-component $B_{y}$ of the magnetic field in increasing $T_{c}$ is much stronger than that of the $x$-component, $B_{x}$. For theAFM case however (see Figures $7 d-7 f$ ), $T_{c}$ is approximately constant for small values of $B_{x}$ and $B_{y}$ located in a domain corresponding to a circular $(\gamma=0)$ or elliptical $(\gamma \neq 0)$ disk centered at the origin in the $\left(B_{x}, B_{y}\right)$-plane. When the magnitudes of $B_{x}$ and $B_{y}$ correspond to the rim of the disk-shaped domains, $T_{c}$ 

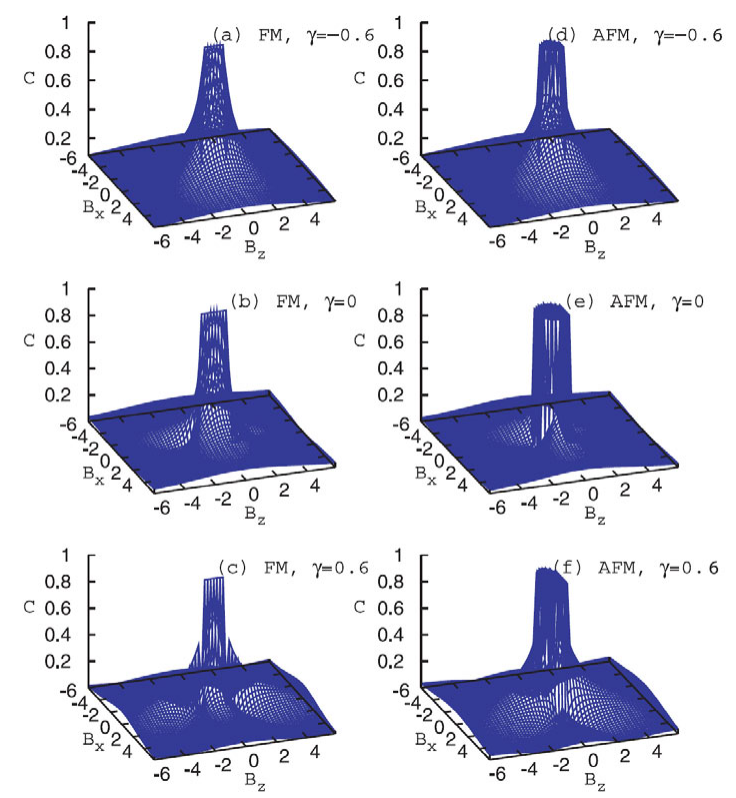

Figure 8. Concurrence of a two-qubit $X Y$ chain in an external magnetic field $\mathbf{B}=B_{X} \mathbf{e}_{X}+B_{z} \mathbf{e}_{z}$ at temperature $T=0$. Results are shown for ferromagnetic (FM) and antiferromagnetic (AFM) two-qubit chains for $|J|=1.0$ and values of the anisotropy parameter $\gamma$ indicated in each figure.

drops sharply and then increases monotonically with both $B_{x}$ and $B_{y}$. The size of this disk-shaped domain decreases with increasing anisotropy parameter. This sharp drop in $T_{c}$ occurs for $B_{x}$ and $B_{y}$ in the vicinity of the values at which the QPT occurs at $T \stackrel{y}{=} 0$ in the AFM case. Therefore, the non-analyticity of $T_{C}$ is also a consequence of the QPT, resulting in a $T_{C}$ that is larger for the FM chain than for the AFM chain.

\section{Case of an external field $B=B_{x} e_{x}+B_{z} e_{z}$}

Many features of the concurrence for this case, such as its invariance under the transformations $B_{x} \rightarrow-B_{x}$ and $B_{z} \rightarrow-B_{z}$, can be deduced from the $\mathbf{B}=B_{x} \mathbf{e}_{x}$ case discussed above and from the study of the $\mathbf{B}=B_{z} \mathbf{e}_{z}$ case in reference [25]. Figure 8 shows the concurrence versus $B_{x}$ and $B_{z}$ for both FM and AFM chains, and for various values of the anisotropy parameter $\gamma$. One sees that for both the FM and AFM cases there is a non-analytic behavior of the concurrence. For the FM case, a non-analyticity occurs only for $B_{x}=0$ when $|\mathbf{B}|=\left|B_{x}\right|=J(1$ $\left.-\gamma^{2}\right)^{1 / 2}$, in agreement with the finding of reference [21] for $\mathbf{B}=$ $B \mathbf{e}_{z}$. As expected from our results in Section 3, the $x$-component of the magnetic field does not induce a QPT in the case of the FM chain, but rather produces a smooth variation of the concurrence as a function of $B_{x}$. The QPT in the FM chain occurs solely because of the $z$-component of the $\mathbf{B}$-field. It appears that the influence of an $x$-component $B_{x}$ in suppressing the QPT outweighs the tendency of the $z$-component $B_{z}$ in inducing a QPT.

For the AFM cases in Figure 8 there are QPTs for all val- ues of the anisotropy and there is always a region of $B_{x}$ and $B_{z}$ in which the concurrence has the maximum value of unity and then drops sharply when critical values of $B_{x}$ and $B_{z}$ are reached. Note that here both components of the B-field lead to QPTs.

\section{Case of an arbitrarily directed magnetic field}

For the case of an arbitrarily directed magnetic field, the eigenenergies are obtained by solving the algebraic equation (10), which can be rewritten as follows:

$$
\begin{aligned}
(E+J)\left[E^{3}-J E^{2}-\left(B_{z}^{2}+\gamma^{2} J^{2}+4 B_{+} B_{-}\right) E\right. \\
\left.+J\left(B_{z}^{2}+\gamma^{2} J^{2}\right)-2 \gamma J\left(B_{+}^{2}+B_{-}^{2}\right)\right]=0 .
\end{aligned}
$$

The analytic solutions to this equation have the following form:

$$
\begin{aligned}
& E_{1}=\frac{1}{3}\left(\frac{a}{2}+2 \frac{b}{a}+J\right), \quad E_{2}=-J \\
& E_{3,4}=\frac{1}{3}\left[\frac{a}{4}(-1 \pm \sqrt{3})+\frac{b}{a}(-1 \mp i \sqrt{3})+J\right]
\end{aligned}
$$

where

$$
\begin{aligned}
a= & \left(8 J^{3}+36 J c-108 d+12 \sqrt{3}\right. \\
& \left.\times \sqrt{27 d^{2}-4 J^{3} d-4 c^{3}-J^{2} c^{2}-18 J c d}\right)^{1 / 3}, \\
b= & J^{2}+3 c, \quad c=B^{2}+\gamma^{2} J^{2} \\
d= & J\left(B_{z}^{2}+\gamma^{2} J^{2}\right)-\gamma J\left(B_{x}^{2}-B_{y}^{2}\right) .
\end{aligned}
$$

Despite the appearance of complex quantities in the equations above (e.g., in Equation (26)), the energies $E_{i}(i=1,2,3,4)$ are real. For the case when the magnetic field is directed along the $Z$-axis, expressions for $E_{i}$ can be reduced easily to the results of reference [25]: $E_{1,2}=\mp J$ and $E_{3,4}= \pm\left(B_{z}^{2}+\gamma^{2} J^{2}\right)^{1 / 2}$. The analytic expressions for the energy eigenstates, $\left|\psi_{i}\right\rangle=\alpha_{i}|00\rangle+$ $\beta_{i}|01\rangle+\gamma_{i}|10\rangle+\delta_{i}|11\rangle(\mathrm{i}=1,2,3,4)$, follow immediately from the system of linear equations defined by the Hamiltonian matrix in equation (9); however, they have rather lengthy expressions and are thus not shown.

In what follows, the direction of the magnetic field, having the magnitude $B$, is characterized by the spherical angles $\theta$ and $\varphi$ in a coordinate frame whose $x$ - and $y$-axes span the XY Heisenberg coupling plane and are directed along the $S_{x}$ and $S_{y}$ coupling components, and whose $z$-axis is orthogonal to the XY coupling plane. In Figures 9-11 we plot and discuss our results as functions of $\theta$ and $\varphi$.

In Figure 9 we show the concurrence of the system at zero temperature (i.e., in its ground state), as a function of the direction of the magnetic field having a fixed magnitude, for both the ferromagnetic and antiferromagnetic couplings. Only cases having nonnegative $\gamma$ are shown (i.e., $J_{x} \geq J_{y}$ ), while the corresponding plots for negative values can be obtained by rotating the coordinate frame in which the magnetic field com- 

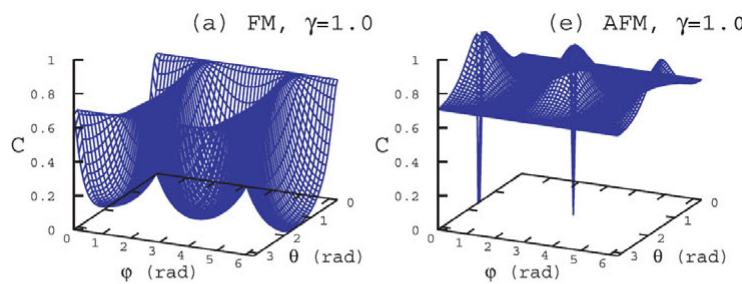

(f) AFM, $\gamma=0.7$
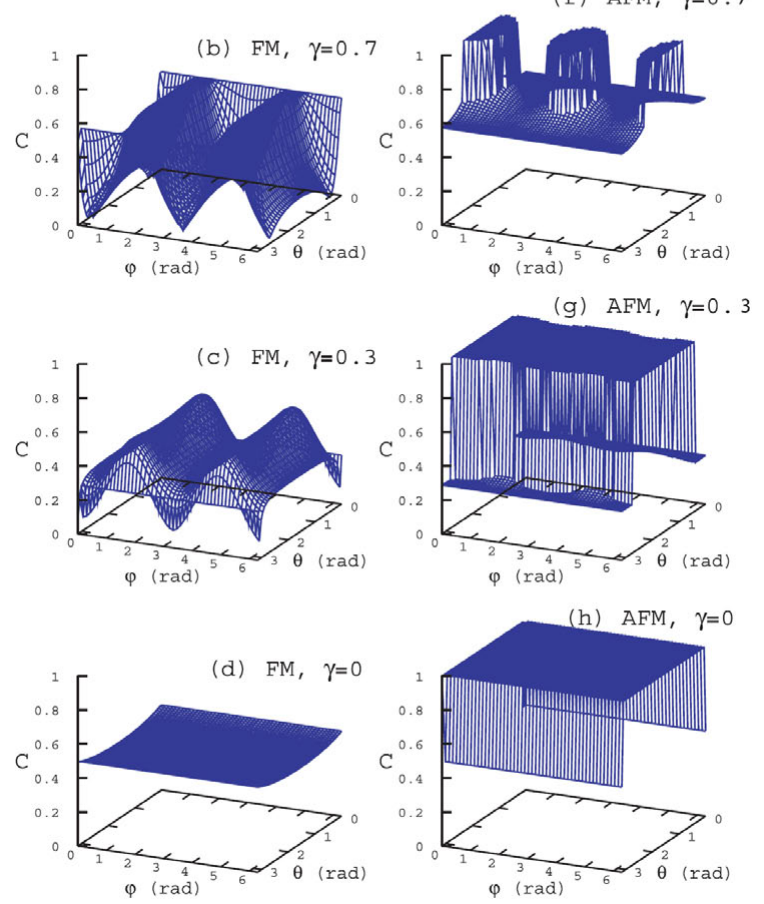

Figure 9. Same as Figure 6, but for an arbitrarily-directed magnetic field, having the magnitude $B=1.0$ and direction characterized by spherical angles $\theta$ and $\varphi$.

ponents are measured by $\pi / 2$ about the $z$-axis (i.e., by making the following substitutions in Figure 9: for $0<\varphi<3 \pi / 2, \varphi \rightarrow$ $\varphi+\pi / 2$, and for $3 \pi / 2<\varphi<2 \pi, \varphi \rightarrow \varphi-3 \pi / 2$ ).

In the case of FM coupling (cf. Figures 9a-9d), the concurrence is a continuous function of $\theta$ and $\varphi$. For FM Ising coupling (cf. Figure 9a) the concurrence is largest when either $\theta=0, \pi$ or when $\varphi=\pi / 2,3 \pi / 2$ (i.e., when $\mathbf{B}$ is parallel to the $z$ - or $y$-axis, respectively), and is zero for $(\theta=\pi / 2, \varphi=0)$ and for $(\theta=\pi / 2, \varphi=\pi)$ (i.e., for $\mathbf{B}$ parallel to the $x$-axis, which for $\gamma=1$ is the Ising interaction axis). For intermediate values of the anisotropy parameter (cf. Figures 9b, 9c), the concurrence is largest when the magnetic field is directed along the axis corresponding to the smaller value of the Heisenberg interaction component. In the limit of an isotropic interaction (cf. Figure 9d), the concurrence exhibits little dependence on $\theta$, and is independent of $\varphi$ due to the cylindrical symmetry of the system.

In the case of AFM coupling (cf. Figures 9e-9h), the concurrence exhibits discontinuities as a function of $\theta$ and $\varphi$, which are due to quantum phase transitions driven by energy level crossings. For the AFM Ising coupling (cf. Figure 9e), as for the FM Ising coupling, the concurrence is zero for $(\theta=$
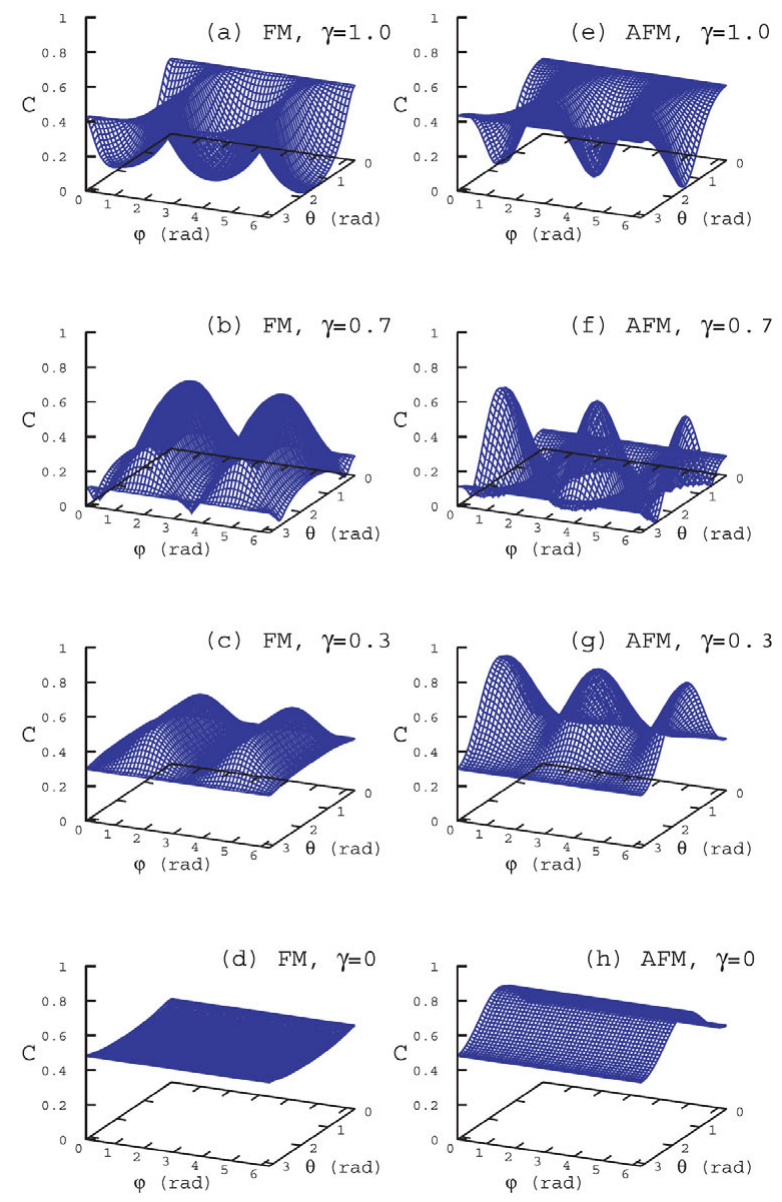

Figure 10. Same as Figure 9, but for nonzero temperature, $k_{B} T=$ 0.25 .

$\pi / 2, \varphi=0)$ and $(\theta=\pi / 2, \varphi=\pi)$, but any infinitisimal deviation from these values results in a quantum phase transition and a jump in the value of the concurrence to unity. Considering results discussed for various $\mathbf{B}$ fields in previous sections, it appears that the occurrence of QPTs for the FM case with $\gamma \neq 1$ is due to the nonzero component of $\mathbf{B}$ along the $z$-axis. For intermediate values of $\gamma$, there are two circle-shaped regions in the $(\theta, \varphi)$ plane for which the concurrence is maximal (unity). The radius of these regions increases as $\gamma$ decreases, and in the limit of isotropic coupling (cf. Figure 9h) the ground state concurrence equals unity for any direction of the magnetic field.

In Figure 10, we present the concurrence of the system's mixed state at nonzero temperature, $k_{B} T=0.25 J$; other parameters are the same as in Figure 9. One sees that for the FM coupling, the effect of nonzero temperature on the concurrence is a general decrease of its magnitude. For the AFM coupling, however, there are qualitative differences in the concurrence behavior as compared to Figure 9. The level crossing-induced quantum phase transitions, which are still present in the results shown in Figures 9e-h, do not result in discontinuities of the concurrence. The discontinuity features present 


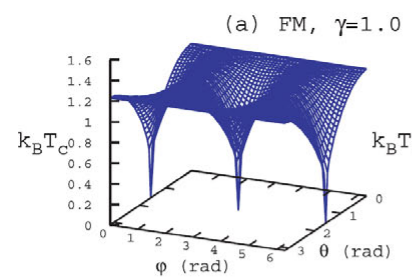

(b) $\mathrm{FM}, \gamma=0.7$

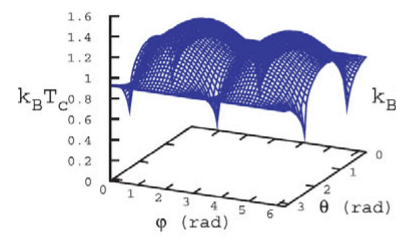

(c) FM, $\gamma=0.3$

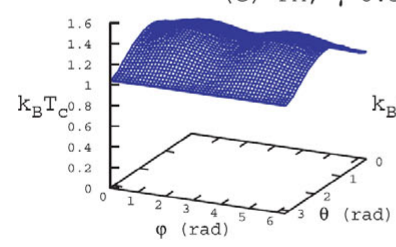

(d) FM, $\gamma=0$

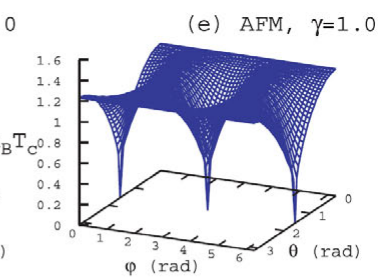

(f) AFM, $\gamma=0.7$

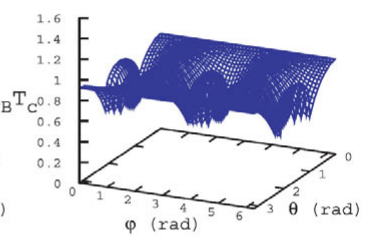

(g) $\mathrm{AFM}, \quad \gamma=0.3$

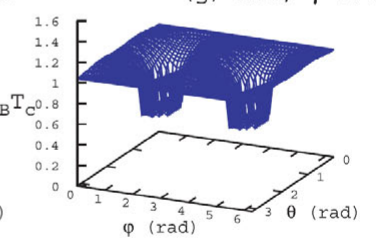

(h) AFM, $\gamma=0$

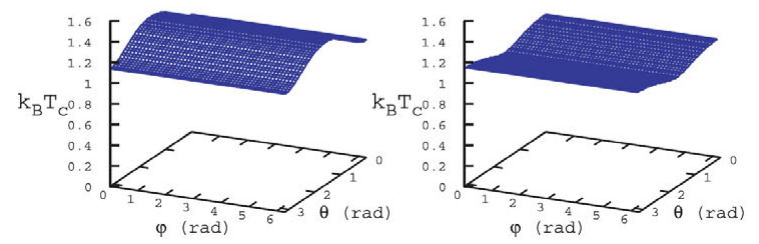

Figure 11. Cut-off temperature, $k_{B} T_{c}$, for FM and AFM couplings for $|J|=1.0$ as function of spherical angles characterizing the direction of an external magnetic field having magnitude $B=2.0$

at $T=0$ (in Figures 9e-9h) are no longer present for $T>0$ (in Figures 10e-10h).

Finally, in Figure 11 we show the numerically calculated cut-off temperature, $k_{B} T_{C}$, at which the concurrence vanishes, as a function of the direction of a magnetic field having a magnitude $B=2.0$. In the case of the FM interaction, the cut-off temperature is highest when the in-plane component of the magnetic field is largest and is parallel to the axis corresponding to the smaller component of the XY coupling. In the case of the AFM Ising coupling (cf. Figure 11e), the behavior of the cutoff temperature is identical to that for the FM coupling (cf. Figure 11a). For the anisotropic AFM coupling, the cut-off temperature has additional local maxima when the magnetic field is directed along the axis corresponding to the greater component of the XY coupling (cf. Figures 11f, 11g). For isotropic coupling (cf. Figure 11h), the cut-off temperature is greatest when the magnetic field is directed along the $z$-axis.

\section{Summary and conclusions}

We have investigated the thermal entanglement of two qubits coupled by an anisotropic Heisenberg XY interaction in the presence of an arbitrarily-directed external magnetic field $\mathbf{B}$ $=B_{x} \mathbf{e}_{x}+B_{y} \mathbf{e}_{y}+B_{z} \mathbf{e}_{z}$. For a better assessment of the influence of each component of the $\mathbf{B}$-field, we start from the simplest cases $\mathrm{B}=B_{x} \mathbf{e}_{x}$ and $\mathrm{B}=B_{y} \mathbf{e}_{y}$, then progressively add one dimension to the $\mathbf{B}$ field by considering $\mathbf{B}=B_{x} \mathbf{e}_{x}+B_{y} \mathbf{e}_{y}, \mathbf{B}=$ $B_{x} \mathbf{e}_{x}+B_{z} \mathbf{e}_{z}$, and finally consider the general case, $\mathbf{B}=B_{x} \mathbf{e}_{x}+$ $B_{y} \mathbf{e}_{y}+B_{z} \mathbf{e}_{z}$. In general, the entanglement, as well as the cutoff temperature above which it vanishes, are found to depend strongly on the anisotropy of the exchange coupling and on the magnetic field strength and orientation.

Specifically, analytical expressions of the concurrence obtained for $\mathbf{B}=B_{x} \mathbf{e}_{x}$ indicate that FM and AFM spin couplings lead to different values of entanglement of the two qubits, and that QPTs due to magnetic field-induced level crossings occur for the AFM case, but never for the FM case. In addition, we find that one can control and produce entangled states of two spins at any temperature and for any anisotropy of the spin coupling by adjusting the magnitude of the $\mathbf{B}$ field. Similar conclusions are drawn for the $\mathbf{B}=B_{y} \mathbf{e}_{y}$ case, whose analytic results can be derived from those for $\mathbf{B}=B_{x} \mathbf{e}_{x}$ by an appropriate transformation. Since both FM- and AFM-coupled qubits lead to the same entanglement for $\mathbf{B}=B_{z} \mathbf{e}_{z}$ [21], it becomes clear that the asymmetry in the entanglement for the FM and AFM cases is due to the existence of a nonzero component of the $\mathbf{B}$ field in the plane of spin coupling.

For $\mathbf{B}$ fields having non-zero components along at least two of the $x$-, $y$-, or $z$-axes, different values of entanglement are found for FM- and AFM-coupled qubits, provided that the B field has a component in the $(x, y)$-plane. In this case also, one can generate entangled states at any temperature and anisotropy by increasing the field strength. In addition, QPTs always occur for the AFM chains, but only occur in FM chains when it is of Ising type or when the $\mathbf{B}$ field has a component along the $z$-axis (a consequence of the occurrence of QPTs in both AFM and FM chains for $\mathbf{B}=B_{z} \mathbf{e}_{z}$ [21]). Our investigations also indicate that higher entanglement is achieved for the XY coupling when the in-plane component of the magnetic field is parallel to the direction in which the exchange coupling between the two spins is smaller.

This work was supported by the Department of Energy, Office of Science, Division of Chemical Sciences, Geosciences, and Biosciences under under Grant No. DE-FG03-96ER14646, and by the Nebraska Research Initiative.

\section{References}

1. A. K. Ekert, Phys. Rev. Lett. 67, 661 (1991)

2. C. H. Bennett, G. Brassard, C. Crépeau, R. Jozsa, A. Peres, W. K. Wootters, Phys. Rev. Lett. 70, 1895 (1993)

3. C. H. Bennett, D. P. DiVincenzo, Nature 404, 247 (2000)

4. F. Verstraete, M. Popp, J. I. Cirac, Phys. Rev. Lett. 92, 027901 (2004)

5. C. H. Bennett, D. P. DiVincenzo, J. A. Smolin, W. K. Wootters, Phys. Rev. A 54, 3824 (1996) 
6. A. Peres, Phys. Rev. Lett. 77, 1413 (1996)

7. V. Vedral, M. B. Plenio, M. A. Rippin, P. L. Knight, Phys. Rev. Lett. 78, 2275 (1997)

8. M. Horodecki, P. Horodecki, R. Horodecki, Phys. Rev. Lett. 80, 5239 (1998)

9. E. M. Rains, Phys. Rev. A 60, 173 (1999); E. M. Rains, Phys. Rev. A 60, 179 (1999)

10. A. F. Abouraddy, B. E. A. Saleh, A. V. Sergienko, M. C. Teich, Phys. Rev. A 64, 050101(R) (2001)

11. D. Loss, D. P. DiVincenzo, Phys. Rev. A 57, 120 (1998); G. Burkard, D. Loss, D. P. DiVincenzo, Phys. Rev. B 59, 2070 (1999)

12. A. Imamoglu, D. D. Awschalom, G. Burkard, D. P. DiVincenzo, D. Loss, M. Sherwin, A. Small, Phys. Rev. Lett. 83, 4204 (1999)

13. R. Raussendorf, H. J. Briegel, Phys. Rev. Lett. 86, 5188 (2001)

14. R. Skomski, A. Y. Istomin, A. F. Starace, D. J. Sellmyer, Phys. Rev. A 70, 062307 (2004)

15. R. Skomski, J. Zhou, A. Y. Istomin, A. F. Starace, D. J. Sellmyer, J. Appl. Phys. 97, 10R511 (2005)

16. B. Georgeot, D. L. Shepelyansky, Phys. Rev. E 62, 3504 (2000); B. Georgeot, D. L. Shepelyansky, Phys. Rev. E 62, 6366 (2000)

17. Y. Makhlin, G. Schön, A. Shnirman, Rev. Mod. Phys. 73, 357 (2001), Appendix A

18. W. K. Wootters, e-print arXiv:quant-ph/0001114; K. M. O’Connor, W. K. Wootters, Phys. Rev. A 63, 052302 (2001)

19. M. C. Arnesen, S. Bose, V. Vedral, Phys. Rev. Lett. 87, 017901 (2001)

20. X. Wang, Phys. Rev. A 64, 012313 (2001)

21. X. Wang, Phys. Lett. A 281, 101 (2001)

22. D. Gunlycke, V. M. Kendon, V. Vedral, S. Bose, Phys. Rev A 64, 042302 (2001)

23. T. J. Osborne, M. A. Nielsen, e-print (1999) arXiv: quant-ph/0202162
24. A. Osterloh, L. Amico, G. Falci, R. Fazio, Nature 416, 608 (2002)

25. G. Lagmago Kamta, A. F. Starace, Phys. Rev. Lett. 88, 107901 (2002)

26. J. S. Pratt, Phys. Rev. Lett. 93, 237205 (2004)

27. M. Asoudeh, V. Karimipour, Phys. Rev. A 71, 022308 (2005)

28. G. F. Zhang, S. S. Li, Phys. Rev. A 72, 034302 (2005)

29. R. Zhang, S. Q. Zhu, Phys. Lett. A 348, 110 (2006)

30. N. Canosa, R. Rossignoli, Phys. Rev. A 73, 022347 (2006)

31. C. X. Li, C. Z. Wang, G. C. Guo, Opt. Commun. 260, 741 (2006)

32. X. H. Peng, J. F. Du, D. Suter, Phys. Rev. A 71, 012307 (2005)

33. D. Mozyrsky, V. Privman, M. L. Glasser, Phys. Rev. Lett. 86, $5112(2001)$

34. A. Sørensen, K. Mølmer, Phys.Rev. Lett. 83, 2274 (1999)

35. Z. C. Kao, J. Ng, Y. Yeo, Phys. Rev. A 72, 062302 (2005)

36. Y. Yeo, T. Q. Liu, Y. E. Lu, Q. Z. Yang, J. Phys. A: Math. Gen. Phys. 38, 3235 (2005)

37. X. Wang, Phys. Rev. A 66, 034302 (2002)

38. Y. F. Wang, J. P. Cao, Y. P. Wang, Phys. Lett. A 342, 375 (2005)

39. S. Hill, W. K. Wootters, Phys. Rev. Lett. 78, 5022 (1997)

40. W. K. Wootters, Phys. Rev. Lett. 80, 2245 (1998)

41. Note that in their investigation of the Heisenberg-Ising case described by the Hamiltonian $H=2 B_{x}\left(S_{1}^{x}+S_{2}^{x}\right)+8 S_{1}^{z} S_{2}^{z}$, the authors of reference [22] mentioned the difficulty of obtaining analytic expressions for the concurrence. However, using the eigenstate basis approach described here, one can derive analytic expressions for the corresponding $\lambda_{i}$ 's.

42. S. Sachdev, Quantum Phase Transitions (Cambridge University Press, 1999); S. Sachdev, Phys. World 12, 33 (1999) 